\title{
Überlegungen zum literarischen Stoff des Telemach
}

\section{Jana Baroková}

This article deals with the literary topic of Telemach. The first attention was aimed at the Austrian children literature at the end of the $18^{\text {th }}$ century. The spread of the novel Telemach reflects the religious and educational framework conditions in Austria, whereby the literary topic of Robinson is corresponding with the German philanthropy. The next chapter deals with François Fénelon and his contribution to the education in his novel Adventures of Telemach. The fourth chapter deals with the reception of the treated literary topic in the Czech literature of the $20^{\text {th }}$ century. The essence of the article is the analysis of the book Telemach und sein Lehrer Mentor by Peter Hacks. A special emphasis is aimed on towards visualizing of the mythical figures and the modernity of the presentation by Peter Hacks. The final chapter deals with the role of the humour in the children and juvenile literature.

Austrian children literature - François Fénelon - literary topic of Telemach - Czech children's literature - Telemach by Peter Hacks - humour in the children's literature - education

Dieser Artikel befasst sich mit dem literarischen Stoff des Telemach. Ich habe mich zuerst der Situation in der österreichischen Kinderliteratur am Ende des 18. Jahrhunderts gewidmet. Die Verbreitung des Werkes Telemach entspricht den konfessionellen und pädagogischen Rahmenbedingungen in Österreich, wobei der literarische Stoff um Robinson mit dem deutschen Philanthropismus korrespondiert. Das nächste Kapitel befasst sich mit François Fénelon und seinem Beitrag zur Erziehung im Roman Die Abenteuer des Telemach. Das vierte Kapitel widmet sich der Rezeption des behandelten literarischen Stoffes in der tschechischen Literatur im 20. Jahrhundert. Den Kern des Artikels bildet die Analyse des Werkes Prinz Telemach und sein Lehrer Mentor von Peter Hacks. Besonderes Augenmerk ist auf die Vergegenwärtigung der mythischen Gestalten und Modernität der Darbietung von Peter Hacks gerichtet. Im letzten Kapitel wird die Bedeutung des Humors für die Kinder- und Jugendliteratur hervorgehoben.

österreichische Kinderliteratur - François Fénelon - Pädagogik - der literarische Stoff des Telemach - tschechische Kinderliteratur - Telemach bei Peter Hacks - Humor in der Kinderliteratur

\section{Einführung}

In meinem Artikel befasse ich mich dem Werk Telemach und sein Lehrer Mentor (1997) von Peter Hacks. Der spätantike literarische Stoff, den P. Hacks adaptiert hat, gehört im Rahmen der literaturgeschichtlichen Forschung zu den Meilensteinen, durch die sich in den Epochen der Aufklärung und der Romantik die Entwicklung der deutschen und österreichischen Kinder- und Jugendliteratur zu trennen beginnt. Der literarische Stoff wurde in den vorigen Jahrhunderten auch in der tschechischen Literatur rezipiert; im zwanzigsten Jahrhundert ist in diesem Zusammenhang das Werk Staré recké báje a pověsti (1961) von Eduard Petiška (1924-1987) zu nennen. 


\section{Politisches Umfeld der österreichischen Kinderliteratur am Ende des 18. Jahrhunderts}

Im Allgemeinen war die österreichische Kinderliteratur am Ende des 18. Jahrhunderts in den Händen des katholischen Klerus. Ernst Seibert (2005) arbeitet drei Thesen auf, die den Ausgang dieser Entwicklung in der österreichischen Monarchie prägen. Erstens herrschte in der Zeit der Aufklärung eine Spannung zwischen katholischer und protestantischer Pastoraltheologie. Das Kindheitsbild erhält gegenüber dem protestantischen Kindheitsbild ein eigenständiges Profil. Zweitens wird in der österreichischen Kinder- und Jugendliteratur das Verhältnis von Herrscherhaus und dem Volk mitreflektiert. Drittens verläuft der Prozess der Emanzipation unter den konfessionellen und pädagogischen Rahmenbedingungen, die in Österreich bestimmend sind, im Vergleich zu Deutschland langwierig und schwerfällig. Ernst Seibert fasst zwei Kontroversen zusammen, und zwar die Auseinandersetzung zwischen dem Jansenismus und dem Philanthropismus auf der philosophischklerikalen Ebene und die zwischen Telemach und Robinson auf der Ebene der kinderliterarischen Werke. Der Jansenismus war (v. a. in den Niederlanden und in Frankreich) im 17. und 18. Jahrhundert eine Bewegung innerhalb der katholischen Kirche, die eine Rückkehr zu Lehre und Kultus des frühen Christentums anstrebte. In Österreich ist der Jansenismus als innerkatholische Polarisierung zu verstehen, die sich gegen die Macht der Jesuiten wandte. Diese innerkatholische Reformbewegung war bemüht, die Kindererziehung zu beeinflussen. Aufgrund der allgemeinen Schulpflicht wurden nun auch Kinder zu Lesern von Lesestoffen, die stark durch die jansenistische Pädagogik geprägt waren. (vgl. Seibert 2005: 98)

\section{François Fénelon und sein Beitrag zur Erziehung}

Nach Ernst Seibert wurde die österreichische Robinsonaden-Literatur in den jugendliterarischen Bearbeitungen auf der Ebene der Trivialliteratur rezipiert, wo vor allem Motive der Bewährung und Welterfahrung der Protagonisten zu finden waren. Dagegen zeigt das Thema des Telemach - verfasst von dem Franzosen François Fénelon - ein völlig anderes pädagogisches Modell. Während Robinson Crusoe auf dem Meer das Abenteuer sucht und sich vom väterlichen Haus und vom Einfluss des Vaters entfernen möchte, sucht Telemach auf der Schifffahrt seinen Vater und bringt ihn letztendlich nach Hause.

François deSalignacdelaMothe-Fénelon(1651-1715) stammtaus einerverarmten Familie des gehobenen Provinzadels. Nach dem Studium am katholischen Seminar wurde er zum Priester geweiht. In seiner seelsorgerischen Tätigkeit versuchte er die Rigorosität des Katholizismus zu mildern. Trotzdem lehnte er den protestantischen Glauben ab. Auf Empfehlung eines vorgesetzten Bischofs wurde er zum Erzieher des Enkels und Erben Ludwigs XIV., des Herzogs von Burgund, ernannt. Als Pädagogen 
und Psychologen gelingt es ihm „[...] seinen schwierigen Zögling, der zum Jähzorn und zur Unbeherrschtheit neigt, zu einem sanften, selbstbestimmten und moralisch integren Menschen zu erziehen“" (Saage 1998: 67). In seinem Abenteuerroman über Telemach (1699) wollte er seinen Zögling auf unterhaltsame Weise belehren. Er wählte die Gattung des Fürstenspiegels, eines damals beliebten didaktischen Genres, in dem das Verhalten sowie die Aufgaben und Pflichten eines Herrschers anschaulich thematisiert werden. Inhaltlich schöpfte er dabei aus der griechischen Mythologie, konkret aus Homers Odyssee. In Fénelons Roman unternimmt Telemach in Begleitung von Minerva, der Göttin der Weisheit, die in der Verkleidung des Lehrers Mentor erscheint, eine abenteuerliche Schifffahrt und begibt sich auf die Suche nach seinem Vater Odysseus. Fénelons höchstes Ziel war es dabei, durch die Gestalt und die pädagogische Wirkung des Mentors dem jungen Thronfolger die Kunst des Regierens und der gerechten Herrschaft beizubringen. Mentor verurteilt Prunk und Herrschsucht, hebt vor allem die Ideale des humanistischen Denkens hervor und plädiert stets für das Wohl des Volkes. König Ludwig XIV. fühlte sich jedoch durch diese indirekte Kritik an seiner Person als Herrscher bedroht und verbannte F. Fénelon aufs Land (vgl. Schikorsky 2003 15).

\section{Die literarische Figur des Telemach in der tschechischen Literatur}

Einst war der pädagogische Roman Die Abenteuer des Telemach in der europäischen Jugendliteratur ein sehr beliebtes Buch. Allein ins Tschechische wurde es insgesamt dreimal übersetzt: Unter dem Titel Př́hody Telemachovy war es im Jahr 1796 in der Übersetzung von Josef Javůrek, im Jahr 1815 in der Übersetzung von Josef Liboslav Ziegler und zuletzt im Jahr 1888 in der Übersetzung von Jan Ev. Hulakovský erschienen (vgl. Cipro 1991).

Unten befasse ich mich mit keiner der oben erwähnten Übersetzungen, weil diese bereits als stark veraltet gelten können. Gegenstand der weiteren Analyse soll nur das Werk von Eduard Petiška sein, obwohl auch dieses schon vor mehr als fünfzig Jahren erschienen ist.

Im Werk Staré recké báje a pověsti (1961) von Eduard Petiška wird Telemach als Sohn des Odysseus zwar an einigen Stellen des Buches erwähnt, wie z. B. in den Sagen Gýges a prsten (S. 176) und Odysseovy cesty (S. 202), dem Helden selbst ist jedoch keine abgeschossene Sage, die seinen Namen tragen würde, gewidmet. Zunächst handelt Telemach nicht selbständig, für sich selbst, sondern wird nur als geliebter Sohn von Odysseus dargestellt. So will z. B. in der Sage Gýges a prsten Odysseus nicht in den Krieg ziehen, täuscht lieber vor den Boten des Volkes Wahnsinn vor und widmet sich dem Ackerbau auf seinen Feldern. Erst als einer der Boten Telemach vor das Pferdegespann des Königs legt, hebt dieser den Sohn auf und zieht mit den anderen in den Krieg. 
Auch in der Sage Odysseovy cesty verwendet Eduard Petiška wieder die auktoriale Erzählweise und schildert Telemach bereits als einen tapferen jungen Mann, der sich während der Schifffahrt nach den weisen und erfahrenen Ratschlägen der Göttin Athene richtet, um seinen gefangenen Vater Odysseus zu finden:

Pallas Athéna se vznesla na Olymp a Telemachos poslechl její rady. Opustil s mnoha dary krále Meneláa a jeho manželku Helenu a vyrazil na moře. Vyhnul se mořské úžině, kde číhala lod’s ženichy, a za př́iznivého větru štastně doplul k břehům Ithaky. (Petiška 1961: 223)

Am Ende dieser Sage wächst Telemach aufgrund seiner abenteuerlichen Seefahrten zu einem entschlossenen jungen Mann und Kämpfer heran, der selbständig entscheidet und sogar seiner eigenen Mutter Penelope Anweisungen erteilt:

”O tom, kdo smí vzít luk do ruky, rozhoduji jenom já,“ vmísil se do sporu Telemachos. „Zbraně patří mužům. Jdi, matko, do své komnaty a nevycházej z ní." [...] Telemachos se opásal mečem, chopil se kopí a postavil se otci po bok. (Petiška 1961: 233)

Wir sehen, dass im Werk Staré recké báje a pověsti von Eduard Petiška Prinz Telemach einer der Protagonisten ist, die der pädagogischen Intention von François Fénelon, dem Autor des französisch verfassten Werkes Die Abenteuer des Telemach (um 1695), völlig entsprechen. Telemach(os) stellt in Petiškas Fassung jedoch nur eine der im Rahmen der griechischen Sagen und Mythen handelnden Figuren dar. Im ursprünglichen Bildungsroman von F. Fénelon, der an das vierte Buch der Odyssee anknüpft, begibt sich Telemach auf die Suche nach seinem Vater Ulysses, wobei er von Mentor begleitet wird, hinter dem sich Minerva, die Göttin der Weisheit verbirgt. Während der Schifffahrt durch Ägypten, Phönizien, Zypern und Kreta gerät der Held in verschiedene kriegerische Ereignisse und politisch angespannte Situationen, die er mithilfe von Mentor schlagfertig oder auch diplomatisch löst. In Mentor hat Telemach einen kompetenten Lehrer, der ihm alle Tugenden eines Herrschers beibringt und ihn von Tyrannei und Eroberungssucht bewahrt. Damalige Leser schätzten an diesem Werk vor allem das sittlich begründete politische Denken und die Forderung nach einer ethischen Handlungsweise der Herrscher, die Kriege verhindern könnte. Auf der formalen Ebene zeigt sich der erzieherische Aspekt des Werkes in der Modellbeziehung „Erzieher und Zögling“. Bei diesem Modell wird davon ausgegangen, dass Tugend automatisch durch äußeren Erfolg belohnt wird. Als ein weiterer erzieherischer Aspekt fungiert in den erzieherischen Romanen die Warnung des Mentors vor Gefahr und Bedrohung. Oft wird der Protagonist auf die Probe gestellt, um so die Erfahrung zu machen, dass tugendhaftes Handeln zu Glückseligkeit und Zufriedenheit führt.

In der Adaption von E. Petiška fehlt die Figur des leitenden und belehrenden Mentors, auch die Göttin der Weisheit Minerva selbst tritt hier nicht auf. 


\section{Prinz Telemach und sein Lehrer Mentor von Peter Hacks}

Das Werk Prinz Telemach undsein Lehrer Mentor des deutschen Dramatikers, Erzählers, Lyrikers und Kinderschriftstellers Peter Hacks (1928-2003) verarbeitet die alte Göttersage mit viel Witz, Humor und Ironie und wandelt so den literarischen Stoff - auch mithilfe der Illustrationen von Uwe Häntsch - in eine gegenwartsbezogene Abenteuergeschichte um. Das Buch enthält 22 Kapitel, deren Überschriften eindeutig die Aufmerksamkeit des Lesers fesseln sollen, wie z. B. Lehrer wie Raupen (S. 5), Die hundertzwanzig Hochzeiter (S. 15), Die Eigenheiten der Greise (S. 55) oder Heimfahrt der Pustebacke (S. 115). Darüber hinaus deuten sie unmittelbar an, dass es sich um humorvolle Geschichten handelt. Die humoristische Darstellung der Handlung der einzelnen Sagen und Mythen kennzeichnet das ganze Buch. Der Humor und der Witz lassen sich den in direkter Rede gehaltenen Aussagen entnehmen. So spricht zum Beispiel im ersten Kapitel der Hirte der Palastschweine - der königliche Saumeister Triefauge Eumel - zu den Lehrern, die auf der Suche nach einem passenden Lehrer, dem Mentor für den Prinzen von Ithaka sind:

„Ich bitte euch“, sagte der Hirt der Palastschweine, „tretet ein mir zum Gefallen. Die leeren Ställe machen mich traurig. Ich vermisse das gutmütige Quieken und Grunzen meiner verlorenen Kinder; ich will euch unterbringen und mir vorstellen, die alten Bewohner seien zurückgekehrt."

„Wir sollen dir deine Sauen und Eber ersetzen?“ sagten die Lehrer empört.

„In einer Weise bin ich verstanden“, sagte Triefauge Eumel. „Aber ihr riecht nicht so gut".

(Hacks 1997: 9-10)

François Fénelon schrieb seinen Roman als eine praktische pädagogische Anweisung für einen aufgeklärten künftigen Herrscher, der aufgrund seines selbstlosen Verhaltens, seines gerechten Handelns und seiner tapferen Taten von allen Untertanen geliebt wird. Er bearbeitete dabei den literarischen Stoff und die Motive der griechischen Göttersagen. In der Adaption von Peter Hacks entsteht ein Anachronismus, der in der zeitgeschichtlichen Seite des Werkes erscheint. Der erwachsene Leser wird sich als Mitleser und Erzieher bei der Lektüre vielleicht an bestimmte kulturgeschichtliche Fakten erinnert fühlen und dabei seine Kenntnisse der griechischen Mythologie auffrischen, der junge Leser wird sich dagegen vor allem über die grotesken Schilderungen der Abenteuer und die frechen Kommentare der Protagonisten amüsieren. Hier sieht der junge Leser Telemach als einen zwar unerfahrenen aber dennoch selbstbewussten Jüngling, den der Mentor während der gefährlichen Schifffahrt durch seine Weisheit zähmt und vor den überall lauernden Gefahren schützt. 


\section{Vergegenwärtigung der mythischen Gestalten und Modernität in der Darbietung von Peter Hacks}

In diesem Abschnitt widme ich mich einigen Protagonisten, wie sie in der Fassung von Peter Hacks auftreten und handeln. Anhand kurzer Charakteristiken und ihrer in direkter Rede bzw. in Kommentaren dargestellten Verhaltensweisen ist die moderne Auffassung der Figuren und die Bemühung von Peter Hacks um eine parodistische Erzählweise gut zu erkennen.

Telemach, Prinz von Ithaka, ist der elfjährige Sohn des Königs Odysseus. Er ist mit dem königlichen Hirten der Palastschafe befreundet. Er ist sich bewusst, dass er bei der Suche nach seinem Vater einen guten Berater benötige. Seine Bitte an die Mutter Penelope, ihm einen passenden Lehrer zu besorgen, wird jedoch von den Heiratskandidaten, die sich um Telemachs Mutter bemühen, als Frechheit und Ungezogenheit abgelehnt. Bei der Vorstellung der Lehrer-Kandidaten handelt Telemach selbständig und verabschiedet sich zornig von allen drei Heiratskandidaten, die behaupten, dass sein Vater tot sei. Er bleibt fest überzeugt, dass er einen guten Lehrer finden wird.

Minerva, die Göttin der Weisheit, verwandelt sich in Mentor, weil sie die Unfähigkeit der künftigen Lehrer Telemachs ratlos macht:

„Wer bist du, Mensch, und woher kommst du?“ grollte Jupiter. [...] „Ich heiße Mentor“, sagte er. „Ich bin ein Lehrer“.

„Ein Lehrer“, sagte Jupiter, „und wagst dich in den Kreis der Götter?“

„Ein Lehrer fürchtet die Götter nicht“, sagte der Mann. [...] „Er ist im Besitz des Wissens und also der Macht". (Hacks 1997: 31)

Mentor stellt sich Telemach zuerst als ein dreister und unansehnlicher Bewerber auf die Lehrerstelle vor, gewinnt jedoch die Sympathie des Prinzen dadurch, dass er als erster der Kandidaten auf dessen Frage, ob sein Vater noch am Leben sei, positiv antwortet. Er zögert jedoch nicht - falls nötig - eine veraltete erzieherische Methode an dem Prinzen zu verwenden:

„Bewege sofort deine Zunge, Plattfuß“, sagte Telemach.

Mentor watschelte ziemlich flink heran und langte sich Telemach am Schlafittchen. Er legte ihn übers Knie. Er zog ihm sein königliches Kleid über dem dürren Hintern stramm, holte den Rohstock aus dem Gewand und - (Hacks 1997: 34)

Mentor trägt immer ein Frettchen, familiär Fretty genannt, in der Tasche seines Mantels, dessen Name Schulz angeblich die Abkürzung für Schulzeit bedeutet. Das Tier tritt als sein Berater auf, oft wird es jedoch wegen seiner unpassenden Aufrichtigkeit und Offenheit von Mentor gescholten. Ein Andermal tritt das Frettchen in der Rolle des Lieblingstieres seines Herrn auf: 
Er hatte sich das Frettchen um den Hals gelegt, wie einen Kragen. (Hacks 1997: 35)

Neptun wird als großer König der Meere vorgestellt. Seine anfänglich majestätische Gestalt wirkt im weiteren Verlauf der Erzählung allmählich kleiner, unbedeutender und schließlich einfach lächerlich:

Zwischen den Inseln Pale und Same kam ein großer Mann aus dem Meer gegangen. Das Salzwasser rann ihm von Haupt und Schulter, während das Meer seine Beine umrauschte und umschwoll. Er lagerte sich am Ufer, gestützt auf den Ellenbogen des rechten Arms. [...] Da sein Leib noch triefte, bildete sich um ihn eine Pfütze. In seinem Haupthaar rannten noch Krabben hin und her. Er verströmte einen heftigen Fischgeruch. (Hacks 1997: 40)

Im Gespräch mit seinem Neffen, König Antinous, handelt Neptun schlagfertig und nüchtern sachlich, anstatt ihm das vorgetäuschte Interesse zu glauben:

Antinous fuhr fort:

„Und wie geht es der Tante Göttin?“

„Sag schon endlich, was du willst“, sagte Neptun. (Hacks 1997: 41)

Zum König Nestor führt Telemach seine jüngste Tochter Polykaste. Der einstige große Herrscher ist nun ein alter schläfriger Greis. Trotzdem verhandelt er mit Telemach sachlich und nüchtern, und der jugendliche Scharfsinn seiner Rede verunsichert Telemach am Anfang. Schließlich kann er Nestor jedoch genauso witzig, schlagfertig und entschieden zurückweisen:

„Weisester König, ältester Mensch, wissensmächtiger der Fürsten“, redete er ihn an, „außerordentlicher Nestor von Pylus ... “

„Wie kannst du glauben“, unterbrach ihn Nestor, „ein Herrscher von meiner Erfahrung könnte auf derart schale Schmeicheleien hereinfallen?“

„Man hat mich gelehrt“, entschuldigte sich Telemach, „wenn sie vom hohen Alter ganz vertrottelt sind, hören sie wieder auf sowas“. (Hacks 1997: 63)

Polykaste, die Tochter des Königs Nestor, führt Telemach samt Mentor in die Tanzgrotte Kalypsowelt, die der Göttin Kalypso gehört. Das Verhalten dieser Göttin ist sehr selbstbewusst und modern; sie wundert sich, dass zusammen mit Telemach dessen Lehrer zum Tanzabend kommt. Auf ihren Einwand hin kontert Telemach schlagfertig wie folgt:

„Du bist das erste Kind“, sagte Polykaste zu Telemach, „das zu Kalypso seinen Lehrer mitnimmt“. „Ich bin das erste Kind“, sagte Telemach, „das sich seinen Lehrer ausgesucht hat". (Hacks 1997: 73)

Der Wind wird personifiziert und handelt als ein Protagonist. Auf Befehl der Göttin Salzmarie legen sich die Winde und Stürme hin, wenn es die Gefahr gibt, dass 
Telemach ertrinkt. Einer der Winde führt mit Telemach ein Gespräch über seine verwandten Winde:

Der Wind, wie man hört, lispelte. Er redete ganz anständig, nur bei den S-Lauten gab er zu viel Luft.

„Wer", sagte Telemach, „sind die anderen?"

„Der Dicke“, sagte der Wind bereitwillig, „issst unssser Aufssseher, Pussstebacke. Der da drüben issst der Wessstwind, der der Sssüdwind, der der Ossstwind und jener ..."

„Der Nordwind“, fiel ihm Telemach in die Rede.

Der Küstenwind war von seinem Wissen beeindruckt. (Hacks 1997: 98)

Die oben angeführte Charakterisierung der handelnden Figuren zeigt, dass die Protagonisten des Werkes in der Auffassung von Peter Hacks in alltäglichen Situationen als moderne Menschen handeln: humorvoll, dynamisch und lebenslustig, keineswegs wie die berühmten Gestalten der griechischen Sagen und Mythen.

\section{Rolle und Bedeutung des Humors in den Kinderbüchern}

An dieser Stelle möchte ich einige Worte zur Bedeutung des Witzes und Humors in der Kinderliteratur hinzufügen.

Es ist schwer, die verschiedenen Formen des Humors - sei es Witz, Satire, Ironie oder Sarkasmus - auf einen gemeinsamen Nenner zu bringen. Oft wird der Humor als eine innere Kraft bezeichnet, die den Menschen durch das Lachen befreit und ihm den erwünschten Abstand von der Realität der Welt - aber auch von sich selbst - ermöglicht. Wesentliche Merkmale des Humors sind heitere Gelassenheit und ein liebevoller Ernst, die der Nüchternheit des Alltags widerstehen. Es besteht eine Varietät der Formen und Stufen, die vom närrischen, naiven, sentimentalen, optimistischen und pessimistischen bis hin zum tragischen Humor reicht (vgl. Bausinger 1970: 7). Vor allem ist die Frage zu beantworten, inwieweit der Humor in der Kinder- und Jugendliteratur seinen Platz hat, wenn er als eine spezifische Fähigkeit und eine geistige und seelische Kraft an den erwachsenen, reifen Menschen gebunden ist. Eine unumstrittene Voraussetzung bildet hier die legitime Erwartung, dass junge Menschen reif genug sind, um Witz und Humor in der Literatur zu begreifen.

Eine weitere Frage ist, inwieweit die Jugendliteratur Wegbegleiter und Wegweiser in Richtung auf ein Verständnis des Humors sein kann. Eine wichtige Feststellung betrifft die Bedeutung und Funktionen des Humors in der Kinderund Jugendliteratur. Dabei soll mit dem Begriff „Humor des Kindes“ gearbeitet werden. Die unumgängliche Konsequenz des Humors ist das Lachen des Kindes über das komisch Geschilderte, also eine Fähigkeit, die bei der Erziehung eindeutig zu fördern ist. Die komisch, witzig, mit Humor geschilderte Realität wird mithilfe der Sprache artikuliert. Als wahre Meister der humorvollen Kinderliteratur gelten eindeutig Erich Kästner, Paul Maar und Christine Nöstlinger, deren Kunst des 
Humors sich auf der Skala von einer liebenden Redeweise voll von mildem und sanftem Witz durch lustvolles Umsetzen der Bedeutung der Wörter und komische Wirkung der Sprachspiele bis zur scharfsinnigen Satire, zerstörerischen Ironie und frechem Sarkasmus bewegt.

\section{Schlusswort}

Im oben analysierten Werk Prinz Telemach und sein Lehrer Mentor von Peter Hacks sind meiner Meinung nach nicht nur humoristische Elemente zu finden, sondern im Ganzen erhält das Werk die Form einer Parodie auf die alten griechischen Sagen und Mythen. Telemach ist zwar auf der Suche nach seinem Vater Odysseus, jedoch die Abenteuer, die er mit seinem Lehrer Mentor erlebt, gehören eher in die Sphäre des sprachlichen Witzes und der Übertreibung als in den Bereich der abenteuerlichen Queste. Die Handlung der einzelnen Geschichten ist in die Gegenwart eingebettet, wobei die Welt der griechischen Mythologie nur eine äußere Kulisse bildet. Am deutlichsten ist diese Verschiebung der literarischen Gattung in Richtung Parodie in den Kapiteln Blumenmädchen (S. 71) und Kalypsowelt (75) zu beobachten. Polykaste führt Telemach in eine Diskotheke, die die Göttin Kalypso betreibt. Als Anachronismus erscheint also die zeitgeschichtliche Seite des Werkes. Der erwachsene Leser wird der Geschichte mit kulturgeschichtlichem Interesse gegenübertreten und die humoristische und parodistische Erzählweise als anachronistisch empfinden. Dem jungen Leser dagegen werden vor allem die grotesken Abenteuer der Protagonisten Spaß machen.

\section{Primärliteratur:}

Hacks, Peter (1997): Prinz Telemach und sein Lehrer Mentor. Berlin, Eulenspiegel Verlag. Petiška, Eduard (1961): Staré recké báje a pověsti. Praha, Albatros Verlag.

\section{Sekundärliteratur:}

Bausinger, Hermann (1970): Humor in der Kinder- und Jugendliteratur. Bericht über die XVI. Internationale Jugendbuchtagung (3. Mainau-Fortsetzungstagung) vom 1.-8. April 1970 in Urach (Württemberg). Insel Mainau, Angelos. S. 7-33.

Cipro, Miroslav (1991): Fénelon a výchova. K 300. výročí vydání příběhů Telemachových. Prag.

Doderer, Klaus (Hg.) (1984): Lexikon der Kinder-und Jugendliteratur. Weinheim und Basel, Beltz.

Saage, Richard (1998): Utopie als „Fürstenspiegel“. Zu Fénelons „Die Abenteuer des Telemach“. In: UTOPIE kreativ 95/1998. S. 66-77.

Schikorsky, Isa (2003): Kinder-und Jugendliteratur. Köln, Literatur und Kunst.

Seibert, Ernst (2005): Kindheitsmuster in der österreichischen Gegenwartsliteratur. Frankfurt am Main, Peter Lang. S. 95-110.

Wild, Reiner (Hg.) (1990): Geschichte der deutschen Kinder-und Jugendliteratur. Stuttgart, J. B. Metzlersche Verlagsbuchhandlung. 